\title{
Evaluación mediante actigrafía de la efectividad de la luminoterapia sobre el sueño en personas mayores con demencia institucionalizadas
}

\author{
N. Cibeira ${ }^{\mathrm{a}}$, A. Maseda ${ }^{\mathrm{a}}$, J.L. Rodríguez-Villamil ${ }^{\mathrm{a}}$, L. Lorenzo-López ${ }^{\mathrm{a}}$, A.X. Pereiro ${ }^{\mathrm{b}}$, \\ J.C. Millán-Calenti ${ }^{\mathrm{a}}$ \\ ${ }^{a}$ Universidade da Coruña, Grupo de Investigación en Gerontología, Instituto de Investigación Biomédica de A \\ Coruña (INIBIC), Complexo Hospitalario Universitario de A Coruña (CHUAC), SERGAS, A Coruña, España \\ ${ }^{b}$ Universidade de Santiago de Compostela, Departamento de Psicoloxía Evolutiva e da Educación, Santiago de \\ Compostela, A Coruña, España
}

Objetivos: La luminoterapia es una terapia no farmacológica efectiva en manifestaciones conductuales de las demencias. El objetivo de este estudio fue conocer a través de registros mediante actigrafía el efecto de la luminoterapia sobre el sueño en personas con demencia moderada-severa institucionalizadas.

Métodos: Estudio piloto de tipo longitudinal, comparativo y prospectivo. Participaron 11 usuarios de un complejo gerontológico especializado en demencias ubicado en A Coruña. Los participantes se dividieron aleatoriamente en dos grupos: experimental y control. El grupo experimental recibía sesiones diarias de luminoterapia de $30 \mathrm{~min} /$ día en la franja horaria 10:30-12:30, de lunes a través de registros con actígrafos colocados en la muñeca de cada persona, durante 5 días antes (pre) de la intervención, y 5 días después de finalizar (post).

Resultados: De los parámetros proporcionados por los registros de actigrafía se analizaron los principales indicadores de calidad del sueño: eficiencia, latencia e índice de fragmentación del sueño. No se encontraron diferencias significativas entre los grupos de estudio, ni cambios significativos a lo largo del tiempo (pre vs post) en el grupo de luminoterapia en ninguno de los tres parámetros analizados. Sin embargo, analizando cada caso de manera individual, se encuentra que en el caso de un sujeto con presencia de trastornos del sueño previos al inicio de la intervención sí se encuentran diferencias estadísticamente significativas en el tiempo en cuanto a la eficiencia del sueño $(\mathrm{p}=0,037)$, siendo el tamaño del efecto grande $(\mathrm{d}=-1,78)$.

Conclusiones: Se muestra evidencia preliminar de la efectividad de la luminoterapia para la mejoría de los trastornos del sueño en personas con demencia, mientras que en las personas sin presencia de alteraciones del sueño previas al inicio de la intervención no se encontraron efectos significativos. Son necesarios futuros estudios de investigación con muestras más grandes y con participantes con presencia de alteraciones o trastornos del sueño previo al inicio de la intervención para comprobar la eficacia de la luminoterapia sobre el sueño.

Este trabajo ha sido financiado por la Xunta de Galicia (ED431C2017/49 y Redes FrailNet IN607C 2016/08 y REGIDEM IN607C2017/02). 